\title{
CRISPR/Cas9 genome editing in wheat
}

\section{Authors: Dongjin Kin, Burcu Alptekin, and Hikmet Budak}

The final publication is available at Springer via http://dx.doi.org/10.1007/s10142-017-0572-x

Kim, Dongjin, Burcu Alptekin, and Hikmet Budak. "CRISPR/Cas9 genome editing in wheat."

Functional and Integrative Genomics (September 2017): 1-11. DOI: 10.1007/s10142-017-0572-x.

Made available through Montana State University's ScholarWorks

scholarworks.montana.edu 


\title{
CRISPR/Cas9 genome editing in wheat
}

\author{
Dongjin Kim $^{1}$ • Burcu Alptekin ${ }^{1} \cdot$ Hikmet Budak $^{1}$ \\ 1 Cereal Genomics Lab, Department of Plant Sciences and Plant \\ Pathology, Montana State University, Bozeman, MT, USA
}

\begin{abstract}
Genome editing has been a long-term challenge for molecular biology research, particularly for plants possess complex genome. The recently discovered Clustered Regularly Interspaced Short Palindromic Repeats (CRISPR)/ CRISPR-associated protein 9 (Cas9) system is a versatile tool for genome editing which enables editing of multiple genes based on the guidance of small RNAs. Even though the effi-ciency of CRISPR/Cas9 system has been shown with several studies from diploid plants, its application remains a challenge for plants with polyploid and complex genome. Here, we applied CRISPR/Cas9 genome editing system in wheat proto-plast to conduct the targeted editing of stress-responsive transcription factor genes, wheat dehydration responsive element binding protein 2 (TaDREB2) and wheat ethylene responsive factor 3 (TaERF3). Targeted genome editing of TaDREB2 and TaERF3 was achieved with transient expression of small guide RNA and Cas9 protein in wheat protoplast. The effec-tiveness of mutagenesis in wheat protoplast was confirmed with restriction enzyme digestion assay, T7 endonuclease as-say, and sequencing. Furthermore, several off-target regions for designed sgRNAs were analyzed, and the specificity of genome editing was confirmed with amplicon sequencing. Overall results suggested that CRISPR/Cas9 genome editing system can easily be established on wheat protoplast and it has a huge potentiality for targeted manipulation of wheat genome for crop improvement purposes.
\end{abstract}

\section{Introduction}

Plant genome editing aiming to generate more yielded and resilient varieties has always been a challenge. Thus far, several methods such as EMS mutagenesis and T-DNA insertions have been utilized to create random mutations; however, these methods do not provide a solution for targeted genome editing (Belhaj et al. 2015). Advances in technology promote the discovery and utilization of genome editing methods such as zinc finger nucleases (ZFNs) and TAL effector nucleases (TALENs) which enable the editing of a gene of interest in a precise manner. However, design and construction of gene editing via these technologies have been problematic and considerably expensive since protein engineering is required for the editing of the gene of interest (Gaj et al. 2013; Bortesi and Fischer 2014). Recently, an important tool for precise genome editing, Clustered Regularly Interspaced Short Palindromic Repeats (CRISPR)/CRISPR-associated protein 9 (Cas9), was discovered which is relatively easy to use and more cost-effective compared to other methods, and it has a potential to change plant improvement strategies in a revolutionary manner.
CRISPR/Cas9 system is a versatile tool for genome editing where multiple genes can be targeted based on the guidance of small RNAs (Doudna and Charpentier 2014). It is the key component of bacterial and archaeal adaptive immunity which was initially discovered in Escherichia coli in the 1980s (Ishino et al. 1987). However, the astonishing function of CRISPR/Cas9 system remained elusive until it was found that Streptococcus thermophilus can acquire resistance against a bacteriophage by integrating a 
genome fragment of an infectious virus into its CRISPR locus (Barrangou et al. 2007). In this new system, destruction of target DNA relies on a double-stranded break which is guided by a crRNA transcript (Schiml and Puchta 2016). Following the targeted DNA breakage, there are two types of DNA repair mechanisms which can be activated: nonhomologous end joining (NHEJ) or homology-directed repair (HDR). NHEJ is an error-prone mechanism resulting in imperfect repair and causes the interruption of gene function. On the other hand, HDR uses a template for the repairing process and generates perfectly repaired new DNA (Schiml et al. 2014; Belhaj et al. 2015). NHEJbased repairing mechanism may also cause nonspecific mutations which arises from possible off-targeting effect of designed sgRNAs on other regions in the genome. In order to increase the specificity of RNA-guided targeted mutagenesis and decrease the off-targeting, protein engineering methods were applied for editing Cas9 nuclease and Cas9 mutants. For example, Cas9D10A (Cas9 nickase variant) has been utilized for more precise editing of genomes (Ran et al. 2013).

Following the discovery of CRISPR/Cas9 system, several genome editing studies for plants have been performed both in monocots and dicots such as Nicotiana benthamiana (Li et al. 2015), Nicotiana tobaccum (Gao et al. 2014), Arabidopsis thaliana (Li et al. 2014), Oryza sativa (Shan et al. 2014), and Sorghum bicolor (Jiang et al. 2013). Even though genome editing via CRISPR/Cas9 system has certain advantages, there are several pitfalls which make its application troublesome for some plant species. For instance, the polyploid nature of several crop species increases the possibility of off-target mutations and decreases genome editing specificity (Peng et al. 2015). Additionally, the editing of each copy of a gene inside the genome is another controversial issue particularly for genes which possess a high copy number in several genomic locations. Bread wheat (Triticum aestivum L.) which is an important crop providing more than $20 \%$ of daily calorie intake for humans has a complex genome structure formed with the combination of three different genomes: A, B, and D (Ling et al. 2013; Choulet et al. 2014). The hexaploid nature of the wheat genome makes this plant an important model for studying and optimizing the genome editing system. In this study, we applied CRISPR/Cas9 genome editing system for two abiotic stress-responsive transcription factor genes, wheat ethylene responsive factor 3 (TaERF3) and wheat dehydration responsive element binding protein 2 (TaDREB2) in wheat protoplast. The targeted mutagenesis generated with CRISPR/ Cas9 was confirmed with restriction enzyme digestion assay, $\mathrm{T} 7$ endonuclease I assay, and sequencing. The possible effect of off-targeting by designed sgRNAs was analyzed with in silico methods, and targeted editing of gene of interest was proven with amplicon sequencing. Current findings indicate that targeted editing of genes in polyploid plant genomes can be accomplished via CRISPR/Cas9 system where a combination of in silico off-target proofing and NGS can be used for improvement of genome editing specificity.

\section{Materials and methods}

\section{In silico analysis of target genes and generation of sgRNA}

For conducting CRISPR/Cas9-based genome editing in wheat protoplast, the full complementary DNA (cDNA) sequences of TaDREB2 (GenBank ID DQ353852.1) and TaERF3 (GenBank ID EF570122.1) were retrieved from NCBI nucleotide archive (https://www.ncbi.nlm.nih.gov/nucleotide/). The cDNA sequences were mapped to wheat genome assembly (v1) (provided by IWGSC) by utilizing Blast tool kit (Camacho et al. 2009), and the exon-intron boundaries were determined for each gene. In addition, each copy of the genes of interest located on different sub-genomes was analyzed in terms of their homology to each other at both sequence and protein levels. Small guide RNA (sgRNA) sequences were chosen, and associated oligos were manually designed based on GenBank sequences for each target gene (Table 1, Supplementary Figs. 1 and 2). Chosen sgRNAs were also analyzed with blasting against three different genomes of wheat: $\mathrm{A}, \mathrm{B}$, and $\mathrm{D}$, to determine the editing ability of sgRNAs in different sub-genomes.

\section{Cloning of target-specific sgRNA oligo into Cas9 vector}

For cloning of target-specific sgRNAs, the protocol from Shan et al. (2014) was followed. The cloning was performed with utilizing pTaU6-gRNA plasmid which contains the wheat TaU6 promoter with a specific guide RNA cloning site and guide RNA scaffold (Shan et al. 2014). Followed by the design and synthesis of sgRNAs for targeted gene editing, the sgRNAs were sub-cloned into pU6-gRNA plasmid from BbsI enzyme site (NEB \#R3539) after sgRNA annealing. For this ligation, synthesized oligos for target-specific sgRNAs were designed with overhangs, 5'-GTTGN(20)-3' in the forward oligo and $5^{\prime}$-AAACN $(20)-3^{\prime}$ in the reverse oligo, complimentary to BbsI enzyme site in pTaU6-sgRNA. The annealing of sgRNAs prior to the cloning was performed with $2 \mu \mathrm{l}$ of $10 \times$ buffer (NEB CutSmart buffer $10 \times$ ) and $9 \mu$ of each $10 \mu \mathrm{M}$ oligo pairs. The sgRNA annealing mixture was denatured at $95{ }^{\circ} \mathrm{C}$ for $5 \mathrm{~min}$, and the annealing was realized with a gradual temperature decrease $\left(1{ }^{\circ} \mathrm{C}\right.$ per minute) to $25^{\circ} \mathrm{C}$. Subsequent to annealing, the sgRNA was ligated with BbsI-digested pTaU6 vector using T4 DNA ligase (NEB \#M0202). Ligation reaction mix contained 1 $\mu \mathrm{l}$ of T4 ligation buffer, $50 \mathrm{ng}$ of digested vector, $3 \mu \mathrm{l}$ of annealed sgRNA oligos, 2.5 units of T4 DNA ligase, and $\mathrm{ddH} 2 \mathrm{O}$ for a final reaction volume of $10 \mu \mathrm{l}$. The ligation mixture was incubated at room temperature for $1 \mathrm{~h}$ and transformed 
Table 1 The sequences of oligos used in this study

\begin{tabular}{lll}
\hline Primer name & Sequence (5'-3') & Purpose \\
\hline TaERF3_oligo3 & cttgGCGAGGGGCAAGCACTACCG & sgRNA \\
TaERF3_oligo4 & aaacGGTAGTGCTTGCCCCTCGC & sgRNA \\
TaDREB2_oligo1 & cttgGCAGGACGTCGACGAGGACT & sgRNA \\
TaDREB2_oligo2 & aaacAGTCCTCGTCGACGTCCTGC & sgRNA \\
TaU6_F & CCCAAGCTTGACCAAGCCCGTTATCT & Sequencing \\
U6-Spel-F & CGGACTAGTGACCAAGCCCGTTATTCTGAC & Cloning \\
sgRNA-SpeI-R & CGGACTAGTAAAAAAAGCACCGACTCGGTGCCA C & Cloning \\
ERF3_T7_F/R & ACTCCGACGACATGGTCGTCTA/GAATCCATTGCACTTGCGCA & Mutation \\
& TT & validation \\
DREB2_T7_F/R & CCTCCCATTACCACGAGCGA/CCGTGAGCTGCTGCTCATTT & Mutation \\
& & validation \\
TaActin-F/R & TTGCTGACCGTATGAGCAAG/ACCCTCCAATCCAGACACTG & qRT-PCR \\
qDREB2_F1/R1 & CGACGACAAGAAGCGGAAC/TGATCTCCGACACCCACTT & qRT-PCR \\
qDREB2_F2/R2 & GCAAGAAGTCCCGCATCT/CGAGGTCCGGGAAGTAAG & qRT-PCR \\
qERF3_F1/R1 & CGACATGGTCGTCTACGG/TCGAGGAAACCGAAGCAG & qRT-PCR \\
qERF3_F2/R2 & CGACGACTCCGACGACAT/CTTGACGGCGGCAAAGG & qRT-PCR \\
DREB-OffT1-F/R & CCTTCAATGCTGACCCTAGC/AGAAACGAGTGGTAGTACTA & Off-target \\
& TACC & Off-target \\
DREB-OffT2-F/R & CCAAATGAAACAACACAAGGCG/ACCATCATCCTACCGCGCAG & Off-target \\
DREB-OffT3-F/R & CAGCCTTCAATGCTGACCCT/GCTAGAATGTGCTTA ATTGGGGT & \\
& GC & Off-target \\
ERF-OffT1-F/R & GGAGGGCAAGAGGATCATCTT/CCGTCAGCTCTGCATTTGTT & Off-target \\
ERF-OffT2-F/R & GTACTGTAATACATGAGTGAG/AAGCGGATCATCTTCACCAA & \\
\hline
\end{tabular}

into chemically competent cells of a DH5 $\alpha$ strain of E. coli. Transformed cells were spread onto an LB plate containing ampicillin and incubated overnight at $37^{\circ} \mathrm{C}$. Plasmid DNA was isolated using Zyppy Plasmid DNA isolation kit (Zymo Research \# ZD4019), and Sanger sequencing was performed with TaU6-F primer to confirm the successful cloning of sgRNA into the pTaU6 plasmid (Table 1).

For Cas9 construction, the procedure in Shan et al. (2014) was slightly modified from the previously used pJIT1632NLSCas9 vector (Shan et al. 2014). In order to combine the previously cloned pTaU6-sgRNA and Cas9 vector (pJT1632NLSCas9), sgRNA site from pTaU6-sgRNA scaffold vector was sub-cloned into Cas9 vector by PCR amplification (Fig. 1). SpeI-TaU6-F and SpeI-sgRNA-R primers (Table 1) were used for amplification of TaU6 promoter, sgRNA, and gRNA scaffold. The PCR fragment was ligated in the pJTT163-2NLSCas9 from SpeI enzyme site. This final constructed vector containing pTaU6 promotor site, single sgRNA site, and Cas9 sequence was utilized for protoplast transformation.

\section{Protoplast isolation and transformation}

Protoplast transformation was conducted by following the protocol from Shan et al. (2014)). The seedlings of T. aestivum cultivar Chinese spring were grown in 16-h light/8-h dark at $25{ }^{\circ} \mathrm{C}$ for 14 days. Fresh tissues were harvested from 40 to 60 seedlings and sliced into 0.5 -mm strips with a sharp razor blade. The strips were transferred into a petri dish with $0.6 \mathrm{M}$ of mannitol and incubated for $10 \mathrm{~min}$ in the dark for quick plasmolysis. After filtering through nylon meshes, the strips were transferred into a 150 -ml conical flask containing $50 \mathrm{ml}$ of filter-sterilized enzyme solution which contained $20 \mathrm{mM}$ of MES (pH 5.7), $1.5 \%(w / v)$ of Cellulase R10, $0.75 \%(w / v)$ of Macerozyme R10, $0.6 \mathrm{M}$ of mannitol, $10 \mathrm{mM}$ of $\mathrm{KCl}, 10 \mathrm{mM}$ of $\mathrm{CaCl}_{2}$, and $0.1 \%(w / v)$ of BSA. In order to infiltrate the digestion solution into leaf tissues, a vacuum $(380-508 \mathrm{mmHg}$ ) was applied for $30 \mathrm{~min}$ in the dark followed by incubation at room temperature for 5-6 h with gentle shaking (60-80 rpm). Subsequent to enzymatic digestion, $50 \mathrm{ml}$ of the W5 solution containing $2 \mathrm{mM}$ MES (pH 5.7), $154 \mathrm{mM}$ of $\mathrm{NaCl}, 125 \mathrm{mM}$ of $\mathrm{CaCl}_{2}$ and $5 \mathrm{mM}$ of $\mathrm{KCl}$ was added to the conical flask and shaken gently for $10 \mathrm{~s}$ to release the protoplasts. Protoplast cells were collected into three or four $50-\mathrm{ml}$ falcon tubes by filtering the mixture through $40-\mu \mathrm{m}$ nylon mesh and washing the tissue strips on the surface of the nylon mesh three to five times with W5 solution. The tube was centrifuged at room temperature for $3 \mathrm{~min}$ at $80 \times \mathrm{g}$ in a swinging bucket rotor. The supernatant was then removed by pipetting, and the protoplasts were resuspended in $30 \mathrm{ml}$ of W5 solution and placed on ice for $30 \mathrm{~min}$. Without disturbing the protoplast pellet, the supernatant was again removed and the pellet resuspended in $4 \mathrm{ml}$ of MMG solution (4 mM MES ( $\mathrm{pH}$ 5.7), $0.4 \mathrm{M}$ mannitol, and $15 \mathrm{mM} \mathrm{MgCl}$ ) at a final concentration of $10^{6}$ cells per milliliter.

Ten micrograms of plasmid DNA in 10-20 $\mu \mathrm{g}$ was gently mixed with $200 \mu \mathrm{l}$ of protoplasts. Two hundred twenty microliters of freshly prepared PEG solution (40\% ( $w / v)$ PEG 4000, $0.2 \mathrm{M}$ mannitol, $100 \mathrm{mM} \mathrm{CaCl}_{2}$ ) was added and mixed gently by tapping the tube. The mixture was then incubated for $20 \mathrm{~min}$ in the dark. After incubation, $880 \mu \mathrm{l}$ of W5 solution was added to the tube and mixed by inverting the tube to stop the transformation process. The protoplasts were harvested by centrifuging at $80 \times \mathrm{g}$ for $3 \mathrm{~min}$ at room temperature and then 


\section{a Target specific gRNA design}

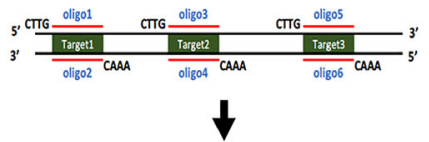

\section{b Annealing of gRNA}

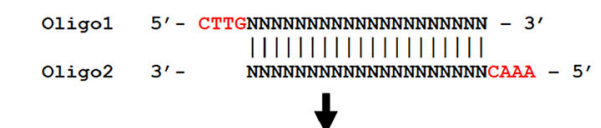

c Cloning gRNA into gRNA scaffold vector

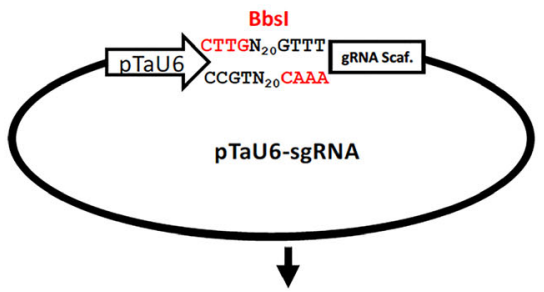

d Sub-cloning into Cas9 Vector

$$
\text { Spel_tau6_F }
$$
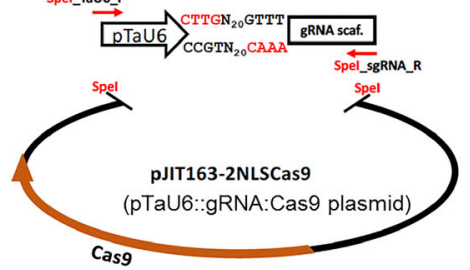

\section{$\downarrow$}

e Transient transformation into wheat protoplasts

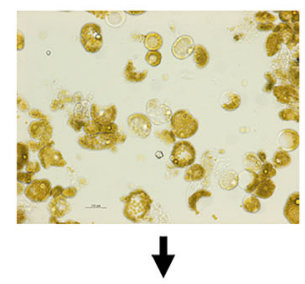

\section{f Verification of target site mutation}

Fig. 1 Schematic description of CRISPR/Cas9-based genome editing application in wheat protoplasts. To begin with, forward and reverse oligos for selected target sites were designed and synthesized in order to insert into the sgRNA scaffold vector (pTaU6-sgRNA) (a). The oligos contained 20-nt target site together with $5^{\prime}$ and $3^{\prime}$ overhangs complementary BbsI digestion sites where the overhangs are $5^{\prime}-\mathrm{CTTGN}(20)-3^{\prime}$ for forward primer and $5^{\prime}$ AAACN(20)-3' for reverse primer. Synthesized oligos were annealed with each other (b), and the paired sgRNAs were ligated into pTaU6-sgRNA plasmid which contained TaU6 promoter and sgRNA scaffold (c). After conformation of sgRNA insertion into pTaU6, sgRNA site from pTaU6sgRNA scaffold vector was sub-cloned into Cas9 vector (pJIT1632NLSCas9), and this combined plasmid was named as pTaU6::gRNA:Cas9 (d). For sub-cloning, TaU6 promoter, sgRNA, and gRNA scaffold were amplified from pTaU6-sgRNA scaffold vector with SpeI-Tau6-F and SpeI-sgRNA-R primers where SpeI enzyme site was used for two component ligations. Thus, the final construction of pTaU6::gRNA:Cas9 was completed, and constructed plasmid was transformed into protoplast (e). The mutation verification was performed with restriction enzyme digestion, T7EI assay, and sequencing (f)

resuspended in $2 \mathrm{ml}$ of W5 solution. To detect the efficiency of wheat protoplast transfection, pGFPGUSplus plasmid (addgene \#64401) which contains GFP expression construct was utilized. Ten micrograms of plasmid vector containing GFP was used for PEG (40\%) mediated transfection which was infected for $20 \mathrm{~min}$. After a 24, 48, and 72-h incubation of transfected protoplast, fluorescence microscopic analyses revealed that approximately 20,40 , and $70 \%$ of transfected protoplasts had GFP expression signal, respectively. The protoplast solution was transferred into 6-well plates, which were then wrapped in aluminum foil and incubated at $23^{\circ} \mathrm{C}$ for 48 $72 \mathrm{~h}$.

\section{Preparation of PCR amplicons for detection of the genome editing events in protoplasts}

Genomic DNA was isolated from the protoplasts using Qiagen DNeasy Plant Mini Kit (Cat. \# 69104) following the manufacturer's protocol. Genomic regions containing the gRNA targets were PCR amplified and subjected to digestion assay and T7 endonuclease I (T7E1) assay for validation of the mutation. PCR amplification was performed in a $25 \mu \mathrm{l}$ reaction volume containing $5 \mu \mathrm{l}$ of protoplast genomic DNA, $2.5 \mu \mathrm{l}$ of primer mix, $0.5 \mu \mathrm{l}$ of $10 \mathrm{mM}$ dNTPs, $5 \mu \mathrm{l}$ of $5 \times$ GC buffer (NEB\# B0519), 3\% DMSO, $0.25 \mu$ of Phusion High Fidelity DNA Polymerase (NEB\# 0530), and ddH2O up to a final volume of $25 \mu \mathrm{l}$ with the following conditions: initial denaturation at $98^{\circ} \mathrm{C}$ for $30 \mathrm{~s}$, denaturation at $98^{\circ} \mathrm{C}$ for $10 \mathrm{~s}$, annealing at $60-72{ }^{\circ} \mathrm{C}$ for $30 \mathrm{~s}$, extension at $72{ }^{\circ} \mathrm{C}$ for $15 \mathrm{~s}$ to 35 cycles, and final extension at $72{ }^{\circ} \mathrm{C}$ for $5 \mathrm{~min}$. PCR products were run on a $1 \%$ agarose gel in TAE buffer, and purification of desired fragments was performed with Zymoclean Gel DNA Recovery Kit (Zymo Research \# ZD4002) for restriction enzyme digestion PCR (RE-PCR) and T7E1 assay.

\section{Restriction enzyme digestion and $\mathrm{T} 7$ endonuclease I assay for validation of genome editing}

In order to detect the mutation at desired restriction enzyme sites, the PCR products were digested with SalI for wheat dehydration responsive element binding protein 2 (TaDREB2) at $37^{\circ} \mathrm{C}$ for $2 \mathrm{~h}$. The PCR fragments containing the gRNA-Cas9 target sites were then amplified by PCR (primer sequences in Table 1), and digested PCR product was analyzed by electrophoresis in $1.2 \%$ agarose gel. So as to identify targeted gene mutation, purified PCR products from the restriction enzyme-digested template were cloned to pMiniT vector (NEB\# E1202). Resulting random colonies were used for plasmid extraction and Sanger sequencing.

For further confirmation of the presence of CRISPR/ Cas9-based mutation at the target site, T7E1 assay was conducted. Firstly, DNA fragments containing the targeted sites were amplified from protoplast genomic DNA using a pair of primers (Table 1) with Phusion High fidelity DNA polymerase (NEB\# 0530). The PCR products were purified 
using Zyppy Plasmid DNA isolation kit (Zymo Research \# ZD4036), and $100 \mathrm{ng}$ of purified PCR product was denatured-annealed under $95^{\circ} \mathrm{C}$ for $5 \mathrm{~min}$ and then ramped down to $15^{\circ} \mathrm{C}\left(10^{\circ} \mathrm{C}\right.$ per minute). Annealed PCR products were then digested with 2.5 units of $\mathrm{T} 7$ endonuclease I (NEB\# M0302) for $1 \mathrm{~h}$ at $37{ }^{\circ} \mathrm{C}$. The T7 endonuclease Idigested product was separated by $1 \%$ agarose gel electrophoresis and used for conformation of mutation in the genes of interest. Digestion efficiency was calculated by measuring band intensities with ImageJ (NIH version 1.5). The gel was isolated, and its intensity measured, with background subtracted. Band intensities were summed to determine total intensities. To calculate the percent of digestion efficiency, the intensity of the non-cleaved band was divided by the total intensity (Shan et al. 2013).

\section{Off-target analysis for CRISPR/Cas9 in wheat}

In order to detect possible off-target potential of designed sgRNAs, specific locations were detected where two mismatched sgRNAs can bind via in silico blast analysis of designed sgRNAs to wheat genome assembly (IWGSC RefSeq v1.0). Based on the blast results, several candidate regions were chosen and oligo pairs were designed to amplify the 400-500 base pair off-target regions which contain the sgRNA target site in the middle (Table 1). The amplification of these regions was performed from both wild-type and mutated protoplast samples. The PCR amplification for all these regions was performed in a $25 \mu \mathrm{l}$ reaction volume containing $5 \mu \mathrm{l}$ of protoplast genomic DNA, $2.5 \mu \mathrm{l}$ of primer mix, $0.5 \mu \mathrm{l}$ of $10 \mathrm{mM}$ dNTPs, $5 \mu$ l of $5 \times$ GC buffer (NEB\# B0519), $3 \%$ of DMSO, $0.25 \mu \mathrm{l}$ of Phusion High Fidelity DNA Polymerase (NEB\# 0530), and ddH2O up to a final volume of $25 \mu \mathrm{l}$ with the following conditions: initial denaturation at $98^{\circ} \mathrm{C}$ for $30 \mathrm{~s}$, denaturation at $98{ }^{\circ} \mathrm{C}$ for $10 \mathrm{~s}$, annealing at 60 to $72{ }^{\circ} \mathrm{C}$ for $30 \mathrm{~s}$, extension at $72{ }^{\circ} \mathrm{C}$ for $15 \mathrm{~s}$ to 35 cycles, and final extension at $72{ }^{\circ} \mathrm{C}$ for $5 \mathrm{~min}$. PCR products were run on a $1 \%$ agarose gel in TAE buffer, and the desired fragment was purified with Zymoclean Gel DNA Recovery Kit (Zymo Research \# ZD4002). The PCR fragments were then tagged with specific primers for amplicon sequencing, and deep sequencing of each amplicon was performed. Obtained deep amplicon sequencing data firstly analyzed with FastQC program to determine the qualified reads prior to analysis. The detected adaptor sequences were removed, and low-quality reads were discarded. The qualified reads were analyzed with two online tools: CRISPR-GA (Güell et al. 2014) and Cas analyzer (Park et al. 2016) (comparison range 70, minimum frequency 5 , WT marker 5). The analysis was performed for wild type and mutated reads separately. The final mutation efficiency for the off-target region was calculated by subtraction of two mutation efficiencies.

\section{Expression analysis of TaDREB2 and TaERF3 by quantitative real-time PCR}

The TaDREB2 and ethylene responsive factor 3 (TaERF3) transcription levels were tested in 7-day-old seeding under short drought treatment $(0,1$, and 4-h dehydration) by using qRT-PCR. RNA was extracted using Rapid Pure RNA Plant Kit (MP Biomedicals Cat\# 112722000), and $2 \mu \mathrm{g}$ of RNA was treated with DNase I (Sigma Cat\# SLBR4100) and reverse-transcribed to cDNA using ProtoScript II first-strand cDNA synthesis kit (NEB Cat\# E6560) following the manufacturer's suggestion. Quantitative real-time PCR was performed using a BioRad CFX96 real-time system (C1000 Touch thermal cycler) with two primers per target gene (Table 1). For amplification, iTaq Universal SYBR Green supermix (BioRad Cat\# 172-5121) was used in a final volume of $10 \mu \mathrm{l}$. The cycler was programmed as follows: $95^{\circ} \mathrm{C}$ for $10 \mathrm{~min}$ followed by 40 cycles of $95{ }^{\circ} \mathrm{C}$ for $15 \mathrm{~s}, 60^{\circ} \mathrm{C}$ for $1 \mathrm{~min}$, and then $95^{\circ} \mathrm{C}$ for $15 \mathrm{~s}$. The constitutive gene of Triticum aestivum actin (TaActin) (Yue et al. 2015) was used as internal standard to normalize the transcripts using a gene-specific primer (Table 1). The $2^{-\Delta \mathrm{Ct}}$ method was used to calculate the difference in expression of chosen genes (Livak and Schmittgen 2001).

\section{Results}

\section{Target selection and vector construction for CRISPR/Cas9}

Prior to sgRNA design and vector construction, the genomic region associated with DREB2 and ERF3 was analyzed, and each copy of genes located on A, B, and D genomes was defined. Based on in silico characterization of target genes, DREB2 was defined with three highly similar copies located on $3 \mathrm{~A}, 3 \mathrm{~B}$, and $3 \mathrm{D}$ chromosomes where three homologs of ERF3 were detected on 2A, 2B, and 2D chromosomes. The sgRNA for DREB2 was designed at the beginning of the protein sequence which covers the 8th to 15th amino acids. For ERF3, the target site was located on 143th to 150th amino acid range which was near by the AP2 domain of the protein. Both DREB2 and ERF3 are characterized by the AP2 domain (Mizoi et al. 2012a), and the preference of a different target location on the protein used for testing and characterizing the editing specificity and its association with how far the sgRNA site was from protein domain. Homology analysis of sgRNA to $\mathrm{A}, \mathrm{B}$, and D genome showed that DREB2 sgRNA was not able to edit one copy of gene located in $3 \mathrm{~A}$ chromosome because of two mismatches between designed sgRNA (Table 2). In 2A copy of ERF3, only one mismatch was 
detected in the designed sgRNA which might result in nonediting or decreased editing results.

\section{Transformation efficiency and transient expression of sgRNA and Cas9 protein in protoplast}

The protoplast transient expression system is an effective and simple method to test the specific genome editing capacity of wheat genes via CRISPR/Cas9 system. In this experiment, the final isolated protoplast cells counted as approximately $1 \times 10^{6}$ cells per milliliter (Supplementary Fig. 3 ) which were sufficient for transfection experiments $\left(5 \times 10^{5}\right.$ cells for sample). After 24, 48, and 72-h incubation of transfected protoplast, fluorescence microscopic analyses revealed that approximately 20,40 , and $70 \%$ of transfected protoplasts had GFP expression signal, respectively (Fig. 2). The obtained rate for transformation efficiencies was lower compared to the results of a previous report in rice protoplast system (Xie and Yang 2013), in which 90\% of GFP expression was reported after $72 \mathrm{~h}$ of incubation. However, the obtained efficiencies were sufficient to establish sgRNA and Cas9 expression in wheat protoplast system at the end of $72 \mathrm{~h}$, and the transfection duration was detected as $72 \mathrm{~h}$.

\section{Validation of targeted gene mutation in wheat protoplast}

To test the mutation efficiency of CRISPR/Cas9 system in wheat protoplast, three different methods were utilized. First, RE-PCR assay was used to detect mutations in the target site and to estimate the frequency of mutation as previously described (Gao et al. 2014). Second, the T7E1 assay was used to detect mismatched nucleotides in the target sites and confirm the mutagenesis (Kim et al. 2009). This was tested with mismatch-sensitive T7E1 after melting and annealing, and cleaved DNA fragments would be detected if amplified products contained both mutated and wild-type DNA (Xie and Yang 2013). Finally, Sanger sequencing of the cloned PCR products further confirmed that targeted mutations were introduced at the predicted Cas9 cleavage site, which is three bases upstream of PAM. After a 72-h incubation, the transfected wheat protoplasts were collected for genomic DNA isolation, with protoplasts transfected by pTaU6::sgRNA:Cas9 empty vector as negative control which did not contain sgRNA of target sites. PCR amplification was performed using the primer of target gene-specific oligos (Table 1) which resulted in products that were about $500-700$ bases long. The PCR products of TaDREB2 gene were digested with restriction digestion enzyme Sall which recognizes and digests the target sequence near the PAM site. In TaDREB2 transfection, undigested bands clearly appeared on the samples without Sall. The PCR product of empty vector-transformed protoplasts were completely digested by the Sall enzyme (Fig. 3a), whereas PCR product of TaDREB2 sgRNA/Cas9 transfection digested into expected bands. According to band intensity, the targeting efficiency for TaDREB2 was 50\% (Fig. 3a). Additionally, mutation efficiency for TaDREB2 was detected with T7E1 assay. In this assay, PCR products from genomic DNA from the TaDREB2 sgRNA/Cas9 and empty vector-transfected protoplast were treated with mismatch-sensitive T7E1 after melting and annealing. Cleaved DNA fragments would be detected if amplified products contained both mutated and wild-type DNA. The T7E1-digested fragments were detected in the TaDREB2 genomic DNA but not in the empty vector control (Fig. 3b). The percentage of digestion was about $6.7 \%$ in the TaDREB2. In addition, Sanger sequencing was performed with cloned PCR products to further confirm the presence of targeted mutations at the predicted Cas 9 cleavage site. Sanger sequencing proved that various mutations, including deletion and nucleotide replacement, were detected at the TaDREB2 (Fig. 3c).

T7E1 assay was also performed on the TaERF3 to detect mutation of the target site. The T7E1 digestion fragments were detected in the TaERF3 genomic DNA but not in the empty vector control (Fig. 4a), and the percentage of digestion was about $10.2 \%$ in the TaERF3. In addition, Sanger sequencing results of TaERF3 PCR products were analyzed to confirm the presence of the mutation in the target site. This result showed deletions and changing nucleotides at the targeted region with sgRNAs (Fig. 4b). Overall, these results suggested that the constructed CRISPR/Cas9 vector can be transiently expressed in wheat protoplast and targeted genome editing can be achieved by Cas9/sgRNA complex.

\section{Specificity of CRISPR/Cas9}

In order to detect the target specificity of CRISPR/Cas9-based mutations, three off-target regions for TaDREB2 and two offtarget regions for TaERF3 were chosen with different mismatches compared to sgRNA (Table 3). Amplified PCR products for these regions were sequences with NGS. Amplicon sequencing was resulted with both intended and non-intended mutations which arise from the nature of PCR amplification process. To neglect the effect of non-intended mutations, the mutation rates of wild-type samples was substituted from mutated samples, and the difference was accepted as the offtarget mutation efficiency. Off-target mutation rate was detected for DREB2-OffTarget1 and DREB2-OffTarget3 regions with an efficiency of 0.013 and $0.97 \%$ where the actual targeting efficiency for DREB2 target region was calculated as $6.7 \%$. For DREB2-OffTarget2 region, no off-target effect was detected since the PAM sequence was placed four bases away from the mimicking sgRNA sequence. For those reasons, any off-target effect for ERF3 was not detected for the selected regions. The results confirmed the specificity of Crispr-Cas9-based editing for wheat genome. 
Table 2 Sub-genomic specificity of designed sgRNAs

\begin{tabular}{llllll}
\hline sgRNA name & sgRNA sequence & $\begin{array}{l}\text { Target } \\
\text { gene }\end{array}$ & $\begin{array}{l}\text { Targeted amino } \\
\text { acids }\end{array}$ & $\begin{array}{l}\text { Targeted } \\
\text { genome }\end{array}$ & $\begin{array}{l}\text { Editing } \\
\text { capability }\end{array}$ \\
\hline sgRNA-DREB2 & GCAGGACGTCGACG & TaDREB2 & ESSSTSC & $3 \mathrm{~A}$ & No \\
& AGGACT & & & $3 \mathrm{~B}$ & Yes \\
& & & & $3 \mathrm{D}$ & Yes \\
sgRNA-ERF3 & GCGAGGGGCAAGCA & TaERF3 & VAR G K H Y & 2A & No \\
& CTACCG & & & $2 \mathrm{~B}$ & Yes \\
& & & & $2 \mathrm{D}$ & Yes \\
\hline
\end{tabular}

\section{The TaDREB 2 and TaERF 3 expression under dehydration treatment}

To confirm the role of TaDREB2 and TaERF3 in the genotype used, their expression was investigated under shock drought stress by qRT-PCR. Quantitative gene expression results showed that shock drought treatment stimulated the upregulation of both genes (Fig. 5). The expression of TaDREB2 and $T a E R F 3$ was gradually increased in response to longer incubation of seedlings under dehydrated conditions. These results support the positive regulation of both TaDREB2 and TaERF3 under drought stress.

\section{Discussion}

CRISPR/Cas9 system is an effective method for targeted genome editing, and its efficiency has been shown in several plant species (Gao et al. 2014; Belhaj et al. 2015; Endo et al.
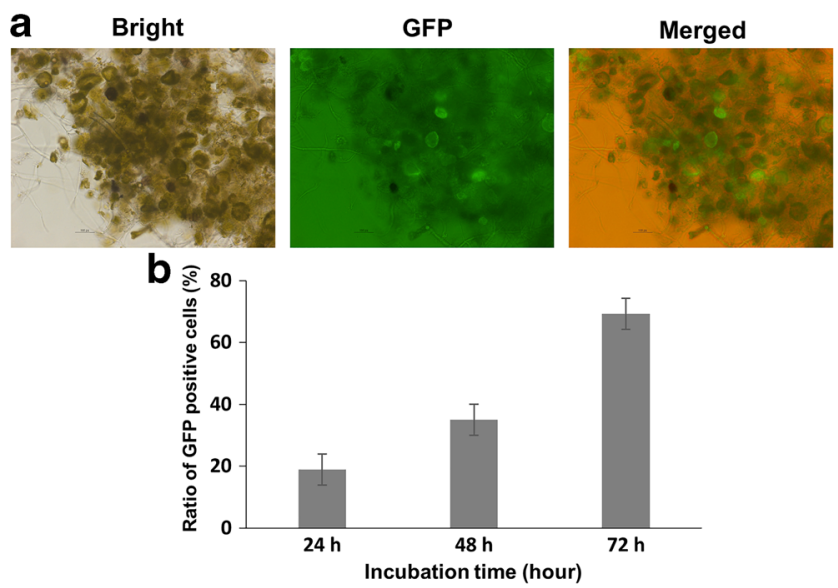

Fig. 2 Transient expression efficiencies for wheat protoplast. a pGFPGUSplus (35S::eGFP) plasmid was transiently expressed in protoplasts to observe the efficient transformation. Part a shows the same protoplast picture with different filters (bright and GFP) where the merged image was obtained from ImageJ (NIH version 1.5). b To detect the transfection efficiency, $10 \mu \mathrm{g}$ of pGFPGUSplus plasmid DNA was used for $1 \times 105$ protoplast cells. The graph shows the ratio of GFPpositive cells to the total number of protoplasts $(n \geq 50)$. The represented mean values are generated based on at least three different transformation results
2015). Although this system is relatively easy to use and more precise compared to other genome editing technologies, there are still some issues, particularly for polyploid plants, such as editing efficiency and off-target mutation rate (Peng et al. 2015). Here, we conducted a series of experiments to show the efficient genome editing with CRISPR/Cas9 system in wheat protoplast. Our results confirmed that CRISPR/Cas9 system is a promising tool for further targeted editing of wheat genome.

Abiotic stress conditions such as drought stress, salt stress, and micronutrient deficiency are serious problems for wheat producers which cause tons of yield loss each year (Araus et al. 2008; Dolferus et al. 2011; Budak et al. 2015). Plants combat against the abiotic stress condition by utilizing a comprehensive signal mechanism where expression of many different genes is involved (Baldoni et al. 2015; Kuzuoglu-
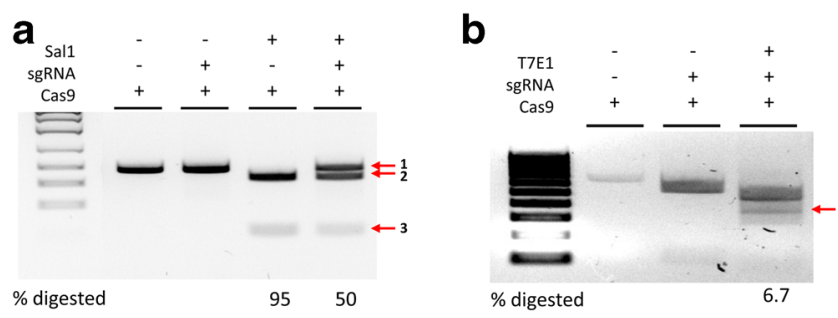

C TaDREB2 $\begin{array}{ll}\text { AGTAGGAGGAAGTGCAGGACGTCGACGAGGACTCGGAGCTCTGCTCGC } & \text { wT } \\ \text { AGTAGGAGGAAGTGCAGGACGTCGACGAG--CTCGGAGCTCTGCTCGC } & -2 \\ \text { AGTAGGAGGAAGTGCAGGACGTCGAC---GACTCGGAGCTCTGCTCGC } & -3 \\ \text { AGTAGGAGGAAGTGCAGGACGTCGACGtAGGACTCGGAGCTCTGCTCGC } & +1\end{array}$

Fig. 3 Mutation detection for TaDREB2. a RE-PCR assay was performed to detect the mutations in TaDREB2 gene from gRNACas9-induced protoplasts. Prior to RE assay, PCR amplification of 600base region around target sites of TaDREB2 was performed. The negative control was an empty vector (without sgRNA but with Cas9) transformed protoplast genomic DNA. Arrows indicate the digested fragments (nondigested band (1), digested long fragments (2), digested short fragments (3)). b Targeted mutations revealed by the T7 endonuclease I (T7E1) assay. The DNA fragments were amplified around the CRISPR/Cas9 target sites by PCR using gene-specific primer from extracted genomic DNA from the protoplast. Mismatches resulting from deletion or insertion at the TaDREB2 PCR amplicon were detected by T7EI digestion. Arrows indicate the digested fragments by T7EI. The ratio of digested DNA band is shown at the bottom of the picture. $\mathbf{c}$ Detection of targeted mutation at the target sites in the TaDREB2 locus was performed with DNA sequencing. The target sequences are marked in blue, and PAM(NGG) motif is marked in red. The numbers on the sides indicate the type of mutations and involved nucleotides 


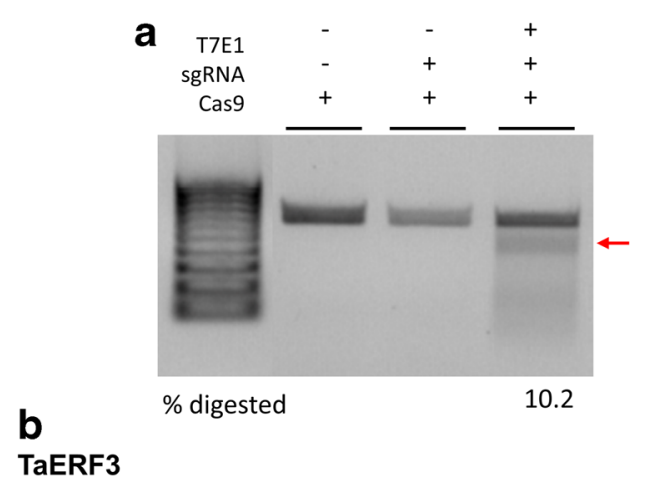

GGCGGCCGCCGCGGTGGCGAGGGGCAAGCACTACCGCGGGGTGCGGCAGC GGCGGCCGCCGCGGTGGCGAGGGGCAAACACTACCGCGGGGTGCGGCAGC GGCGGCCGCCGCGGTGGCGAGGGGCAAGCAC--CCGCGGGGTGCGGCAGC GGCGGCCGCCGCGGTGGCGAGGGGCAAGC----CCGCGGGGTGCGGCAGC

WT

$-2$

Fig. 4 Detection of mutations in the TaERF3. a T7 endonuclease I (T7E1) assay result for TaERF3 is shown. The 600-bp region around target site was amplified by PCR from transformed protoplasts' genomic DNA. The negative control used PCR fragment from empty vector (without sgRNA but with Cas9) transformed protoplast genomic DNA and T7E1 non-treated PCR fragment. Arrows indicate the digested fragments by T7EI. The ratio of digested DNA band is shown at the bottom of picture. $\mathbf{b}$ Detection of targeted mutation at the target sites in the TaERF3 locus was performed with DNA sequencing. The target sequences are marked in blue, and PAM(NGG) motif is marked in red. The numbers at the sides indicate the type of mutations and involved nucleotides

Ozturk et al. 2012). Particularly, transcription factors are keystones for stress responses which affect the expression of many genes by resulting in the activation/deactivation of a comprehensive molecular pathways (Singh et al. 2002). In this study, we selected two important abiotic stress-responsive transcription factor genes, TaDREB2 and TaERF3 which are characterized by the AP2/ERF domain (Mizoi et al. 2012b; Lucas et al. 2011) for performing CRISPR/Cas9-based genome editing application. The effect of DREB genes on increased drought resistance has been shown in several studies (Agarwal et al. 2006; Lata and Prasad 2011; Lucas et al. 2011; Morran et al. 2011). Overexpression of DREB members in Arabidopsis and soybean increased the drought resistance without affecting the growth parameters (Nakashima et al. 2014). In wheat and barley, overexpressed DREB2 and $D R E B 3$ resulted with activated expression of many droughtresponsive genes and provided more drought tolerance (Morran et al. 2011). It was also reported that DREB proteins are more abundant and strongly regulated in response to drought in root tissue than leaf tissue (Lucas et al. 2011). Additionally, in different maize varieties which show differential drought response, a natural variation was detected in $D R E B 2$ promoters which were associated with drought tolerance. On the other hand, ERF3 was defined as an important molecule for root development where its interaction with Wox11 protein causes activation of cytokine response (Zhao et al. 2015). This interaction is further linked with root elongation and root hair development which provide drought resistance (Cheng et al. 2016). In addition, expression of

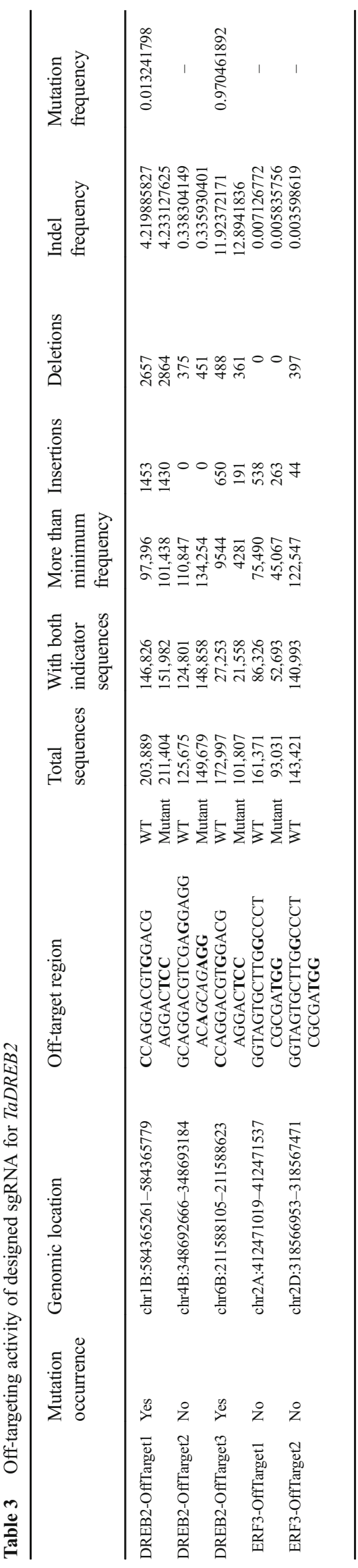


TaDREB2 and TaERF3 showed upregulation in response to shock drought stress in variety Chinese spring (Fig. 5). Overall, these findings indicate that DREB2 and ERF3 are vital genes for abiotic stress tolerance, particularly for drought, and further characterization of functions of these genes is necessary for understanding their involvement in stress response. Regarding this purpose, these genes are thought as good candidates for targeted genome editing where their characterization with stable transformation will provide a deep insight about their functioning in abiotic stress response.

As the first step of CRISPR/Cas9, the target sites with PAM (NGG) sequence in the 3'-region were selected and associated oligos were synthesized. Chosen sgRNAs were inserted into the pJIT163-2NLSCas9 plasmid with a combined TaU6 promoter site. The efficient expression of sgRNA-Cas 9 constructs was obtained for both TaDREB2 and TaERF3. The results showed that rice codon-optimized Cas9 can be efficiently expressed in wheat and utilize for specific genome editing. The transient expression of the sgRNA-Cas9 construct was successfully achieved in wheat protoplast. Interestingly, the transformation efficiency was lower compared to other studies (Shan et al. 2014). This low transformation efficiency might arise from the fragile nature of wheat protoplast as mentioned in some previous works (Sun et al. 2013). In spite of this low efficiency, the obtained transformation rate was sufficient for effective editing of targeted genes in the wheat genome which was confirmed with three different mutation validation techniques. The restriction enzyme digestion assay was only conducted for $D R E B 2$ since there was no defined restriction site in the $E R F 3$ target site. For both genes, the mutation efficiency was also confirmed with the T7EI assay and Sanger sequencing. Combination of three results suggested that mutation efficiency was lower in DREB2 compared to $E R F 3$. This situation might arise from the location of chosen sgRNA site and suggests that target sites which are
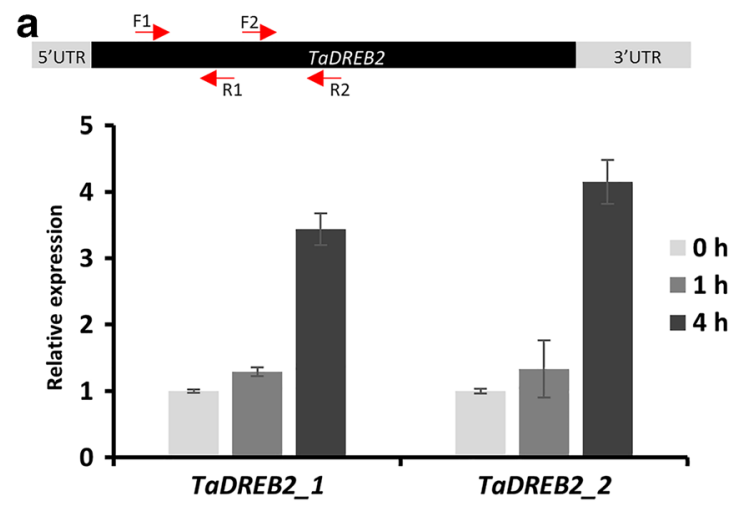

Fig. 5 Expression patterns of TaDREB2 and TaERF3 upon drought treatment. a. Upper panel shows gene model of TaDREB2. Gray boxes are 5' and 3' UTR, and black boxes are exon. Red arrows indicated two different qRT-PCR primers for gene expression analysis. The bottom panel shows the relative expression pattern of TaDREB2 with short drought treatment $(0,1$, and $5 \mathrm{~h}$ of drought incubation). TaDREB2_1 chosen in a close proximity of protein domain work more efficiently compared to further target locations inside the protein.

There are a number of concerns about the specificity of genome editing with CRISPR/Cas9 because of the occurrence of random mutation during the genome editing process (Schaefer et al. 2017; Sharpe and Cooper 2017). In this study, the specificity of genome editing was further investigated with next-generation sequencing of off-target regions which were selected based on the homology of sgRNA to several regions in wheat. Three amplified off-target regions for TaDREB2 were sequenced with amplicon sequencing, and results were analyzed with two different online tools CRISPR-GA (Güell et al. 2014) and Cas analyzer (Park et al. 2016). Results from both programs showed an accordance, however; Cas analyzer results were chosen as representative since the tool enables user to select the range of mutation analysis in given sequences. Furthermore, the minimum frequency parameter in this tool provides a control against randomly occurred mutations which is arisen from the nature of PCR amplification process. In fact, amplicon sequencing results showed the presence of random insertions/deletions in both wild-type and mutant samples which were occurred in the amplification process, probably because of high GC content of the amplicon of interest. Such mutations can easily affect the calculated mutation efficiency rate, particularly in pooled amplicon sequencing for mutation efficiency (Park et al. 2016). In order to avoid this effect of random mutations, the CRISPR mutation efficiency was calculated by subtraction of mutated and wildtype mutation rates. This analysis showed that genome editing efficiency for off-target regions with a real PAM sequence for TaDREB2 was significantly lower compared to targeted sequence. This result highly supports the specificity of genome editing with CRISPR/Cas9 system in wheat even in the presence of some random indels in selected regions. However, the
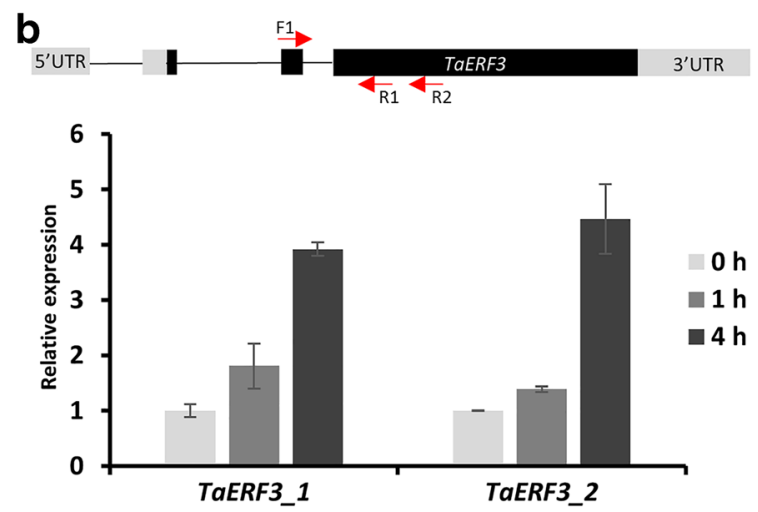

has used qRT-PCR primer F1 and R1, and TaDREB2_2 has used the primers F2 and R2. b. Upper panel shows the gene model of TaERF3. Gray boxes are 5' and 3' UTR; black boxes are exon, and lines are intron. TaERF3_1 has used qRT-PCR primer F1 and R1, and TaERF3_2 has used F1 and R2 primers. The short drought treatment was harvested after 0,1 , and $4-\mathrm{h}$ treatments 
length of such indels was relatively short (one to two bases) which provides support for their randomness.

In this study, the CRISPR/Cas9 genome editing system in wheat protoplast was effectively established. The transient expression of sgRNA and Cas9 protein was performed in wheat protoplast. The genome editing efficiency was shown with restriction enzyme assay, T7 endonuclease assay, and Sanger sequencing. Furthermore, the specificity of editing in wheat was confirmed with amplicon sequencing analysis. Overall, a successful application of CRISPR/Cas9 based genome editing in wheat protoplast was performed with two abiotic stress genes, TaDREB2 and TaERF3. These current findings suggest that CRISPR/Cas9 system is an efficient tool for targeted genome editing for wheat and it has a potential application for manipulation of wheat genome aiming a better crop performance.

Acknowledgements The authors acknowledge Montana Wheat and Barley Committee Grant \#MDA/MWBC CY5416-462 and WinifredAsbjornson Plant Science Endowment. The authors also thank the International Wheat Genome Sequencing Consortium (IWGSC) for prepublication access to the wheat genome RefSeq v1.0.

\section{References}

Agarwal PK, Agarwal P, Reddy MK, Sopory SK (2006) Role of DREB transcription factors in abiotic and biotic stress tolerance in plants. Plant Cell Rep 25:1263-1274

Araus JL, Slafer GA, Royo C, Serret MD (2008) Breeding for yield potential and stress adaptation in cereals. CRC Crit Rev Plant Sci 27:377-412. https://doi.org/10.1080/07352680802467736

Baldoni E, Genga A, Cominelli E et al (2015) Tolerance to drought and salt stress in plants: unraveling the signaling networks. Front Plant Sci 5:1-16. https://doi.org/10.1089/omi.2013.0177

Barrangou R, Fremaux C, Deveau H et al (2007) CRISPR provides acquired resistance against viruses in prokaryotes. Science 315:17091712. https://doi.org/10.1126/science. 1138140

Belhaj K, Chaparro-Garcia A, Kamoun S et al (2015) Editing plant genomes with CRISPR/Cas9. Curr Opin Biotechnol 32:76-84. https:// doi.org/10.1016/j.copbio.2014.11.007

Bortesi L, Fischer R (2014) The CRISPR/Cas9 system for plant genome editing and beyond. Biotechnol Adv 33:41-52. https://doi.org/10. 1016/j.biotechadv.2014.12.006

Budak H, Hussain B, Khan Z, Ozturk NZ, Ullah N (2015) From genetics to fucntional genomics: improvement in doruhgt signalling and tolerance in wheat. Front Plant Sci. https://doi.org/10.3389/fpls.2015. 01012

Camacho C, Coulouris G, Avagyan V et al (2009) BLAST+: architecture and applications. BMC Bioinformatics 10:421. https://doi.org/10. 1186/1471-2105-10-421

Cheng S, Zhou D-X, Zhao Y (2016) WUSCHEL-related homeobox gene WOX11 increases rice drought resistance by controlling root hair formation and root system development. Plant Signal Behav 11: e1130198. https://doi.org/10.1080/15592324.2015.1130198

Choulet F, Alberti A, Theil S et al (2014) Structural and functional partitioning of bread wheat chromosome 3B. Science 345 : 1249721. https://doi.org/10.1126/science. 1249721
Dolferus R, Ji X, Richards RA (2011) Abiotic stress and control of grain number in cereals. Plant Sci 181:331-341. https://doi.org/10.1016/j. plantsci.2011.05.015

Doudna JA, Charpentier E (2014) The new frontier of genome engineering with CRISPR-Cas9. Science 346:1258096. https://doi.org/10. 1126/science. 1258096

Endo M, Mikami M, Toki S (2015) Multigene knockout utilizing offtarget mutations of the CRISPR/Cas9 system in rice. Plant Cell Physiol 56:41-47. https://doi.org/10.1093/pcp/pcu154

Gaj T, Gersbach CA, Barbas CF (2013) ZFN, TALEN, and CRISPR/Casbased methods for genome engineering. Trends Biotechnol 31:397405. https://doi.org/10.1016/j.tibtech.2013.04.004

Gao J, Wang G, Ma S et al (2014) CRISPR/Cas9-mediated targeted mutagenesis in Nicotiana tabacum. Plant Mol Biol 87(1-2):99110. https://doi.org/10.1007/s11103-014-0263-0

Güell M, Yang L, Church GM (2014) Genome editing assessment using CRISPR genome analyzer (CRISPR-GA). Bioinformatics 30:29682970. https://doi.org/10.1093/bioinformatics/btu427

Ishino Y, Shinagawa H, Makino K et al (1987) Nucleotide sequence of the iap gene, responsible for alkaline phosphatase isozyme conversion in Escherichia coli, and identification of the gene product. J Bacteriol 169:5429-5433

Jiang W, Zhou H, Bi H et al (2013) Demonstration of CRISPR/Cas9/ sgRNA-mediated targeted gene modification in Arabidopsis, tobacco, sorghum and rice. Nucleic Acids Res 41:e188. https://doi.org/10. 1093/nar/gkt780

Kim HJ, Lee HJ, Kim H et al (2009) Targeted genome editing in human cells with zinc finger nucleases constructed via modular assembly. Genome Res 19:1279-1288. https://doi.org/10.1101/gr.089417.108

Kuzuoglu-Ozturk D, Cebeci Yalcinkaya O, Akpinar BA, Mitou G, Korkmaz G, Gozuacik D, Budak H (2012) Autophagy-related gene, TdAtg8, in wild emmer wheat plays a role in drought and osmotic stress response. Planta 236(4):1081-1092

Lata C, Prasad M (2011) Role of DREBs in regulation of abiotic stress responses in plants. J Exp Bot 62:4731-4748

Li JF, Zhang D, Sheen J (2014) Cas9-based genome editing in Arabidopsis and tobacco. Methods Enzymol 546:459-472. https:// doi.org/10.1016/B978-0-12-801185-0.00022-2

Li JF, Zhang D, Sheen J (2015) Targeted plant genome editing via the CRISPR/Cas9 technology. Methods Mol Bio 1284:239-55. https:// doi.org/10.1007/978-1-4939-2444-8 12

Ling H-Q, Zhao S, Liu D et al (2013) Draft genome of the wheat Agenome progenitor Triticum urartu. Nature 496:87-90. https://doi. org/10.1038/nature11997

Livak KJ, Schmittgen TD (2001) Analysis of relative gene expression data using real-time quantitative PCR and the $2-\Delta \Delta C T$ method. Methods 25:402-408. https://doi.org/10.1006/meth.2001.1262

Lucas S, Durmaz E, Akpnar BA, Budak H (2011) The drought response displayed by a DRE-binding protein from Triticum dicoccoides. Plant Physiol Biochem 49:346-351. https://doi.org/10.1016/j. plaphy.2011.01.016

Mizoi J, Shinozaki K, Yamaguchi-Shinozaki K (2012a) AP2/ERF family transcription factors in plant abiotic stress responses. Biochim Biophys Acta 1819:86-96. https://doi.org/10.1016/j.bbagrm.2011. 08.004\r10.1016/j.bbagrm.2011.08.004

Mizoi J, Shinozaki K, Yamaguchi-Shinozaki K (2012b) AP2/ERF family transcription factors in plant abiotic stress responses. Biochim Biophys Acta - Gene Regul Mech 1819:86-96. https://doi.org/10. 1016/j.bbagrm.2011.08.004

Morran S, Eini O, Pyvovarenko T et al (2011) Improvement of stress tolerance of wheat and barley by modulation of expression of DREB/CBF factors. Plant Biotechnol J 9:230-249. https://doi.org/ 10.1111/j.1467-7652.2010.00547.x

Nakashima K, Yamaguchi-Shinozaki K, Shinozaki K (2014) The transcriptional regulatory network in the drought response and its 
crosstalk in abiotic stress responses including drought, cold, and heat. Front Plant Sci 5:170. https://doi.org/10.3389/fpls.2014.00170

Park J, Lim K, Kim J-S, Bae S (2016) Cas-analyzer: an online tool for assessing genome editing results using NGS data. Bioinformatics 33:btw561. https://doi.org/10.1093/bioinformatics/btw561

Peng R, Lin G, Li J (2015) Potential pitfalls of CRISPR/Cas9-mediated genome editing. FEBS J n/a-n/a. https://doi.org/10.1111/febs.13586

Ran FA, Hsu PD, Lin CY et al (2013) Double nicking by RNA-guided CRISPR cas9 for enhanced genome editing specificity. Cell 154: 1380-1389. https://doi.org/10.1016/j.cell.2013.08.021

Schaefer KA, Wu W-H, Colgan DF, et al (2017) Unexpected mutations after CRISPR-Cas9 editing in vivo. Nat Methods 14:547-548. doi: $10.1038 /$ nmeth. 4293

Schiml S, Puchta H (2016) Revolutionizing plant biology: multiple ways of genome engineering by CRISPR/Cas. Plant Methods 12:8. https://doi.org/10.1186/s13007-016-0103-0

Schiml S, Fauser F, Puchta H (2014) The CRISPR/Cas system can be used as nuclease for in planta gene targeting and as paired nickases for directed mutagenesis in Arabidopsis resulting in heritable progeny. Plant J n/a-n/a. https://doi.org/10.1111/tpj.12704

Shan Q, Wang Y, Li J et al (2013) Targeted genome modification of crop plants using a CRISPR-Cas system. Nat Biotechnol 31:686-688. https://doi.org/10.1038/nbt.2650
Shan Q, Wang Y, Li J, Gao C (2014) Genome editing in rice and wheat using the CRISPR/Cas system. Nat Protoc 9:2395-2410. https://doi. org/10.1038/nprot.2014.157

Sharpe JJ, Cooper TA (2017) Unexpected consequences: exon skipping caused by CRISPR-generated mutations. Genome Biol 18:109. doi: 10.1186/s13059-017-1240-0

Singh KB, Foley RC, Oñate-Sánchez L (2002) Transcription factors in plant defense and stress responses. Curr Opin Plant Biol 5:430-436

Sun H, Lang Z, Zhu L, Huang D (2013) Optimized condition for protoplast isolation from maize, wheat and rice leaves. Chin J Biotechnol 29:224-234

Xie K, Yang Y (2013) RNA-guided genome editing in plants using a CRISPR-Cas system. Mol Plant 6:1975-1983. https://doi.org/10. 1093/mp/sst119

Yue J, Sun H, Zhang W et al (2015) Wheat homologs of yeast ATG6 function in autophagy and are implicated in powdery mildew immunity. BMC Plant Biol 15:1-15. https://doi.org/10.1186/s12870015-0472-y

Zhao Y, Cheng S, Song Y et al (2015) The interaction between rice ERF3 and WOX11 promotes crown root development by regulating gene expression involved in cytokinin signaling. Plant Cell 27:2469 2483. https://doi.org/10.1105/tpc. 15.00227 